\title{
ROLE OF MONOCLONAL ANTIBODIES AGAINST THE PROGRAMMABLE DEATH (PD-1) RECEPTOR OF T-CELLSIN TARGETED THERAPY OF MELANOMA
}

\author{
Mukantayev K.N., Mukanov K.K., Zhylkibayev A. \\ National Centerfor Biotechnology \\ 13/5, Korgalzhyn road, Astana, 010000, Kazakhstan \\ mukantaev@biocenter.kz.
}

\section{ABSTRACT}

Oncogenic cells have intrinsic properties for evading attack by the immune system, which largely contribute to the oncogenic pathology. Therefore, the development of drugs that can block these properties represents an important goal toward obtaining effective strategies to cure cancer. Currently, monoclonal antibodies targeting the programmable cell death 1 (PD-1) receptor and its ligand (PD-L1) are widely used in targeted therapy, which show high effectivity in clinical studies resulting in a significant increase in the duration of anticancer immunity.

The PD-1/PD-L1 signaling pathway was recently identified as a mechanism of the negative regulation of the immune system, and as the main mechanism by which oncogenic cells evade the body's immune system. Moreover, the expression of PD-1 receptor in the tumour microenvironment cells is related to several other signaling pathways involving both hematopoietic and non-hematopoietic cells. The interaction of PD-1 and PD-L1 inhibits the activity of cytotoxic T-cells and tumour-infiltrating lymphocytes with increased T-cell suppressor activity. Consequently, the PD-1/PD-L1 signaling pathway promotes tumour cells to evade the immune response. Therefore, blockage of PD-1/PD-L1 enhances antitumor immunity, reduces the number of T-cell suppressors and suppresses their activity, and restores the activity of cytotoxic T-cells.

The PD1 receptor and it is ligands PD-L1 and PD-L2 are representatives of the immunoglobulin superfamily and are part of the "immunological control points" system, the main functions of which include the regulation and modulation of the immune response, reduction of immune cell-induced damage in organs and tissues, and prevention of autoimmune processes.

Accordingly, development of therapeutic anti-PD1 and anti-PD-L1 monoclonal antibodies that could lead to the reactivation of a specific antitumor immune response is a promising strategy of tumour immunotherapy. To date, monoclonal antibodies blocking PD-1/PD-L1 have achieved good results and were approved by the Food and Drug Administration.

Keywords: monoclonal antibodies, melanoma, PD1, PD-L1, receptor, immunotherapy of tumours.

\section{INTRODUCTION}

Monoclonal antibodies (mAbs) with the advent of hybridoma technology in 1978 have found wide application in various branches of science and healthcare, including oncology. Interest in hybridoma technology and monoclonal antibodies in the oncology area connected with receiving of drugs capable of affecting various functions of oncogenic cells. Affecting of monoclonal antibodies on the various functions of oncogenic cells is carried out by specific binding of antibodies to proteins of cancer cells and their complexes. In present,50 various type antitumor drugs based on the monoclonal antibodies have produced on the pharmaceutical market.According to literature, a monoclonal antibody to the human epidermal growth factor (HER2) named trastuzumab and several other new HER2 inhibitors increased the life expectancy of HER2 + breast cancer patients (BC). Moreover, monoclonal antibodies to HER2 increased the 5-year survival rate of early-stage breast cancer patients to 90-95\%. Before the appearance of monoclonal antibodies to HER2, breast cancer at any stage was considered to be a malignant neoplasm with an unfavorable prognosis of the disease course [1].

Another monoclonal antibody, which has a stable position in the pharmaceutical market, is Ipilimumab, approved in 2011.At the end of 2014, two new drugs appeared on the market with the same action, namely,Pembrolizumab (the commercial name is KEYTRUDA) and Nivolumab. These monoclonal antibodies are designed for targeted therapy of malignant skin melanoma. The duration of the immune response reached 5 years, after using monoclonal antibodies in $20 \%$ of patients. The traditionally used chemotherapy for skin melanoma had extremely low efficacy,unlike monoclonal antibodies [1].

These monoclonal antibodies are humanized antibodies to programmable cell death 1 (PD-1) receptor obtained in vitro.PD-1 plays an important role in regulating $\mathrm{T}$-cell activity and tolerance of the immune system. There is a positive and negative regulation of T-cell activity. Positive activation of T-cells is a multi-step process. The antigen is processed and exposed on the surface of antigen-presenting cells. The antigen and protein of the major 
histocompatibility complex II MHC are recognized and bound by T-cells using specific (T-cell) receptors. Binding leads to the activation of $\mathrm{T}$-cells and the appearance of their selective copies.Various hormone-like signaling proteins participate in activation of T-cells, called interleukins, which carry out positive stimulation of CD28/CD80 receptors., A multistep cascade is express highly affine IL-2R receptor on T-cells in the presence of a sufficiently strong signal. The result of activation is the clonal expansion of T-cells and the enhancement of their effector functions.

Negative stimulation of T-cells prevents the activation of T-cellsduring the initial interaction of T-cells with antigen presenting cells. One example is CTLA-4 (a protein associated with the cytotoxicity of T-lymphocytes 4) activators of negative stimulation and another one is PD-1 negativestimulation, which is expressed on activated T-, B-lymphocytes, and myeloid cells. Ligands of this receptor, PD-L1, and ODS-2 receptors are exposed on the antigen surface of parenchymal hematopoietic stemcells and certain types of tumor cells. It was found that the PD-L1 receptor is overexpressed in tumor cells [2]. Activation of the receptor by ligands leads to a reduction of interleukins synthesis, respectively to decrease in the immune attack not only to infectious agents but also to certain cancers like malignant melanoma.

These monoclonal antibodies can also be effective against other types of malignant tumors. For example, a positive effect of pembrolizumab was noted in $18 \%$ of patients diagnosed with non-small cell lung cancer, in $27 \%$ of patients with renal cell carcinoma and in $28 \%$ of patients with melanoma. The formation of long-term antitumor immunity was also observed in these patients. Monoclonal antibodies inhibited the growth of various malignant tumors within 20-31 months [3].

In contrast to other cancers, the incidence of malignant skin melanoma increases year by year. In the Republic of Kazakhstan, oncological diseases are the second most commontype of disease. According to news agency News Kazakhstan, about 17 thousand people die each year from the oncological diseases, $42 \%$ of them are of working age. The death rate from cancer in Kazakhstan ranks second in the structure of the population. The annual increase in the number of patients with malignant neoplasms is 5\% [4].

Statistical data show that in Republic of Kazakhstan in 2016, the total number of cancer patients was 160080 people [5]. The breast cancer is $11.6 \%$, lung cancer is $11.4 \%$, skin cancer is $10.7 \%$, stomach cancer is $8.8 \%$, cervical cancer is $4.8 \%$, esophageal cancer $-4.4 \%$, hemoblastosis $-4.4 \%$, colon cancer $-4.4 \%$ and rectum $-4.4 \%$ [6]. It can be seen from the statistics that skin tumors, including melanoma, are one of the most common and difficult to treat forms of cancer in the country. An especially dangerous form of the disease is the metastatic form of the disease since the tumor is resistant to standard chemotherapy and radiation therapy.

However, despite the problems of treating melanoma by traditional methods, malignant skin melanoma cells are susceptible to immunological attack. In this regard, the search for potential targeted therapy is a current research direction. High molecular weight melanoma-associated antigen (HMW-MAA) is considered an attractive target because of its high expression frequency in patients with melanoma, as well as its limited distribution in tissues: it is expressed in $90 \%$ melanoma and tissues with ectodermal, mesodermal and endodermal origin, but it is not found in cells with squamous cell basal carcinoma [7]. Expression of HMW-MAA does not depend on the synthesis of melanin, and also on the primary, ametastatic or recurrent form of the disease. Antibodies caused by it can inhibit the functional properties of HMW-MAA and thus reduce or suppress the metastatic potential of melanoma cells carrying HMW-MAA.

However, despite the positive effect of vaccines, tumor cells have signaling pathways that allow them to evade the immune system. When studying the phenomenon of reducing the anticancer immune response in cancer patients, one of the most important components of the main mechanism was an immunosuppressive signal mediated by the PD-1 receptor ofT cells and its ligand PD-L1 on the surface of tumor cells.

PD-1 was discovered by TasukuHonjo with colleagues from Kyoto University in 1992 [8]. PD-1 expresses on the T-cells and negatively regulates antitumor effect and led to the death T-cells when reacted with PD-L1. In normal physiological conditions, the PD-1 expresses in tonsils cells, human lungs, placental trophoblast, and monocyte, and improves the immunological tolerance of the body. Also, PD-L1 expresses in cells of various tumor pathology of a human such as urothelial cancers, gastrointestinal cancer, lung cancer, breast cancer, melanoma, and ovarian cancer, etc. [8].

Monoclonal antibodies against the PD-1 receptor and its PD-L1 ligand have become important breakthrough in the prevention of cancer. The successful development of PD-modulating drugs has unexpectedly revolutionized the field of oncological immunology and opens the door for the therapy of immune modulation of the tumor, which will have a profound effect on clinical and immunological research in the field of oncology [9].

In this article, we have analyzedthe studies related to the production and use of monoclonal antibodies against the T-cell programmed death receptor (PD-1). On the basis of the literature data, the mechanisms of circumventing cancer cells by anticancer immunity and the prospects for the clinical use of monoclonal antibodies against PD-1 in various malignant tumors in the near future are described.

\section{IMMUNOLOGICAL THERAPY OF CANCER DISEASES}

The immune system plays an important role in the diagnosis and therapy of various human diseases, including oncology. Key factors of effective immune response are specificity, stability, and prevalence of the antigen, by which the immune system recognizes and destroys malignant cells. The immune system recognizes various antigens 
and receptors, which expressed on the membranes of tumor cells causing a cycle of cellular and immunological events, leading to the death of oncogenic cells. However, tumor cells have proteins and receptors on the outer membrane which can inhibit antitumor immune system. Therefore, spontaneous immune responses resulting from the use of low-performance vaccines are not sufficient for regression of tumors [10].

Currently, immunological therapy of many oncological pathologies involves the use of therapeutic vaccines, poly- and monoclonal antibodies, cytokines, immune response inhibitors and adaptive administration of $\mathrm{T}$ cells. In general, immunological therapy of cancer is a complex of different approaches for increasing and prolonging the body's immunological response against cancer [11]. For example, therapeutic vaccines are a mixture of antigens specific for cancer cells, designed to induce an antitumoral immune response. Additionally, inhibitors (anti-cancer antibodies, etc.) of important control points of the anticancer immune response mechanism can be used in conjunction with therapeutic anticancer vaccines. According to several authors, such approaches in the treatment of oncological pathologies can provide long-term disease-free survival and be a promising approach for the complete elimination of certain oncological diseases [12].

According to recent research, vaccines should be used as early as possible to prevent the spread and metastasis of oncogenic cells. In this case, effective vaccines should consist of tumor-associated antigens (TAA), delivered in a way that increases immunogenicity and is combined with adjuvant immunomodulators. As the clinical trials showed, the vaccines proven safe had low toxicity and were effective at low expression levels of the target protein $[13,14,15]$.

It should be noted that TAA is usually overexpressed in oncogenic cells, but can also occur at low concentrations in normal cells. Therefore, the body's immune system produces a certain level of tolerance to these antigens, which can be overcome by applying special types of antibodies aimed at enhancing the immune response. Nevertheless, in the use of vaccines based on TAA, there are certain problems associated with different immunogenicity in different patients, the potential for loss of immunogenicity associated with tumor development, new mutations or antigen differences at different stages of tumor development [16].

The solution of the immune system tolerance problems arising with the use of TAA is the use of a tumorspecific antigen (TSA). Currently, intensive studies are in progress to determine individual TSA capable of eliciting an immune response that is clinically more effective than the immune response from TAA. However, TSA also has a number of certain disadvantages associated with the uniqueness of antigens in the tumor of each individual, while antigens differ depending on the cells of the same tumor site [16]. Despite these problems, in combination with adjuvants, dendritic cells, recombinant viruses, inhibitors of immune system control points and chemotherapy, tumor-associated and tumor-specific antigens have demonstrated their promise in numerous studies.

TAA and TSA were described for various types of oncologic pathology. Antigens associated with breast cancer are the human epidermal growth factor (HER2) receptor, the mucin-1 membrane protein (MUC-1) and the catalytic portion of the human telomerase enzyme- reverse transcriptase (hTERT). The most studied is HER2 from which the E75 vaccine was created. E75 (HER2/neu 369-377a.a) is an immunogenic peptide of the HER2 / neu protein, which is overexpressed in patients with breast cancer. Clinical studies in 186 patients showed the safety of the vaccine, a high immunological response to the peptide and, most importantly, the potential clinical advantage of the vaccine [17]. Immunization with the E75 vaccine in combination with a granulocyte colony stimulating factor (G-CSF) correlated with higher rates of recession-free survival and a lower relapse rate [18].

Vaccines against colon cancer as well as the vaccine against breast cancer were based only on tumorassociated antigens, which could also be expressed on normal cells. Various tumor-associated antigens, such as p53, carcinoembryonic antigen (CEA), MUC1, Sialyl-Tn, 5T4, SART3 and MAGE [19] were used to develop vaccines against colon cancer. Studies have shown that tolerance is a potential barrier to the use of these vaccines, as well as that of breast cancer vaccines. Vaccines were developed to solve the problem and to induce specific T-cell responses that stimulate an immunological response against different TAA and are capable of inducing the complementary reactivity of CD4+ and CD8+ T-cells. In fact, three different strategies have been used to immunize patients using the above-mentioned TAA, Dendritic cells, recombinant viruses and peptide vaccines [19].

In general, these vaccines were not toxic and induced the formation of immunity against p53 [20], CEA [21], 5T4 [22] and EpCAM/KSA [23]. The use of these vaccines not only induced the formation of specific antibodies and T-cells against tumor antigens but also against viral components. Encouraging results were obtained by combining the p53 antigen with overlapping long peptides of p53 [24]. Interestingly, the MVA-5T4 vaccine induced the active form of 5T4-specific antibodies and provided patients with a longer survival time [22].

In case of melanoma, it should be noted that differential antigens or cancer/testis antigens were the focus of tumor-associated antigens studies. Whereas, a mixture of antigens of various classes is usually used in the development of polyvalent vaccines.An important requirement to achieve clinical success with vaccination as a therapeutic strategyis the expression of the target antigen by all the tumor cells of the patient. Human melanoma cells express several antigens that are recognized by autologous and specific T-killers in combination with HLAclass-I molecules. Many of these antigens are suitable targets for tumor immunotherapy since their expression in human melanoma cells is specific and inherentfor this type of cells. Studies have shown that vaccines based on MAGE-1, MAGE-3, BAGE, GAGE-1/2, Melan-A/MART-1 antigens, and tyrosinase can be used as immunological therapy for patients after surgical procedures diagnosed with stage III melanoma, classified in the AJCC manual as a diagnosis with a high mortality risk [25]. 
It should be noted, vaccines based on cancer/testis (CT) antigens were greatlydescribed. Combining of cancer/testis antigens is a group of proteins which was combinedin one group based on their importance in immunotherapy of oncological pathology. In general, the expression of these proteins is limited to male germ cells in adult animals. However, in cancer, these antigens are often re-expressed and,they are often classified as tumor antigens and can serve as targets for the induction of anticancer immunity. CT antigens were identified in melanoma, liver cancer, lung cancer, bladder cancer, and childhood cancer, such as neuroblastoma. In general, the presented results showed that the conducted studies created the basis for immunotherapy as a supplement to traditional cancer therapy. Moreover, the development of cancer vaccines and their use in combination with a traditional cytotoxic therapy or new immunotherapyis an approach with the potential to improve patient survival.

Passive immunotherapy with the use of monoclonal antibodies directed against the target antigens of tumor cells is of interest for the oncological diseases therapy. The effect of monoclonal antibodies is provided by several mechanisms: direct action of an antibody such as blockage of receptors leading to induction of apoptosis or delivery of a cytotoxic agent, immune mechanisms such as complementary cytotoxicity (CDC) or antibody-dependent cellular cytotoxicity (ADCC), and specific effects of these antibodies on the vasculature and microenvironment of the tumor. The most traditional approach of using monoclonal antibodies in oncology is the use of highly specific and highly active antibodies against antigens that are specifically expressed on the surface of tumor cells. Although in theory, this strategy sounds quite simple, the problems lie in search for key antigens that will be neutralized by the $\mathrm{mABs}$ and lead to the complete elimination of the tumor without significant systemic side effects [26].

In the present time, monoclonal antibodies to the programmable cell death 1 (PD-1) receptor presented for immunotherapy directedon the blockade of development control points of the tumor. The PD-1 receptor of T-cells is regulator which can inhibit or completely blockimmunity [26]. In a normal healthy body, the receptor plays an important role in ensuring the effectiveness of the immune system and preventing autoimmunity. For these purposes, in cells under the action of biologically active substances, the receptor PD-L1 is inhibiting the immunological activity. In the oncological organism, biologically active substances secreted by cytotoxic T-cells also lead to the activation of the PD-L1 receptor on the surface of oncogenic cells (figure 1).

Toshio Shimizu et al. (2016) studied the safety, tolerability, pharmacokinetics, pharmacodynamics, immunogenicity, and antitumor activity of Pembrolizumab (mAbs against the PD-1 receptor) in Japanese patients with metastatic solid tumors in Phase I clinical trials. As a result of the studies, no dose of dependent toxic effects was found. Minor side effects of level 1-2 were observed in eighty percent of patients manifested by nausea, malaise, and pyrexia. Side effects associated with the immune system were also at 1-2 levels and were resolved using steroid drugs. Overall, Pembrolizumab at a dose of 2 and $10 \mathrm{mg} / \mathrm{kg}$ was well tolerated in patients with metastatic solid tumors and showed encouraging antitumor activity against melanoma and non-small cell lung cancer [27].

In $\mathrm{Ib}$ phase of the clinical test, authors examined the safety and antitumor activity of Pembrolizumab in Japanese patients with metastatic tumors. In result of conducted research detected 34 patients (81.0\%) experienced side effects associated with treatment. More widespread experienced side effects were itching, maculopapular rash, malaise, and hypothyroidism of 1-2 levels. The drug provided survival at $82.7 \%$ patients with a diagnosis of cutaneous melanoma and at $51.4 \%$ patients with a diagnosis of mucosal melanoma within 12 months. The safety profile of pembrolizumab in Japanese patients was similar to that described in previous clinical studies. Pembrolizumab provided promising antitumor activity in Japanese patients with metastatic melanoma [28].
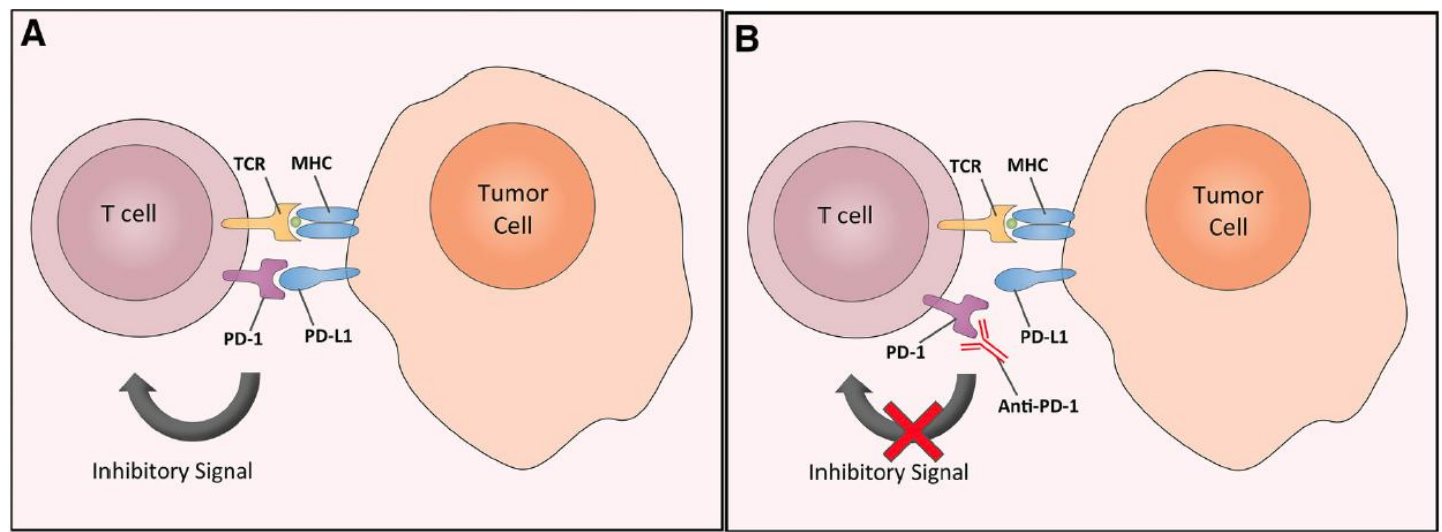

$\mathrm{T}$ cells recognize antigens presented by the major histocompatibility complex (MHC) on tumor cells through interactions with the T cell receptor (TCR). Programmed death 1 (PD-1) is an inhibitory receptor that can terminate or inhibit T cell immune response when interacting with programmed death ligand 1 (PD-L1). A Tumor cells that express PD-L1 can evade immune response by regulating the activity of $\mathrm{T}$ cells through PD-1. B By blocking the PD-1 receptor with an anti-PD-1 mAb, such as nivolumab or MK-3475, T cells are not inhibited by tumor-expressed PD-L1 and are free to mount an immune response against melanoma tumor cells [Mamalis A., Garcha M., Jagdeo J. Targeting the PD-1 pathway: a promising future for the treatment of melanoma. Archives of Dermatological Research, 2014, vol. 306, pp.511-519. doi10.1007/s00403-014-1457-7]

Fig. 1. PD-1's role in inhibiting the immune response through $\mathrm{T}$ cell activity downregulation 


\section{THE ROLE OF PD-1 AND ITS LIGAND PD-L1 IN THE FUNCTIONING OF IMMUNE SYSTEM}

The basic function of PD-1 in normal physiological condition is toinhibitexcessive activity of the immune system in peripheral tissues (autoimmune diseases) by apoptosis of $\mathrm{T}$ cells. In this regard, the signaling pathway associated with the PD-1 receptor plays a key role in the regulation of immunity directed against fungal and viral infections. Studies on mice defective in the CD4 T cell gene and infected with the virus showed that blockage of the PD-1 and PD-L1-inhibitory pathway beneficially affected defective CD8-T cells, restoring their proliferation and expression of cytokines. CD8-T cells acquired the ability to kill infected cells, thereby reducing viral load $[29,30]$.

Tumor cells are capable of inhibiting anti-cancer immunity and evading cytotoxic T-cell attacks by negatively regulating the cytotoxic T-lymphocyte antigen 4 (CTLA-4), mucin 3-chain immunoglobulin T (TIM3), 2B4, attenuator $\mathrm{B}$ and $\mathrm{T}$ lymphocytes (BTLA) and 3 (LAG-3), the programmable cell death 1 receptor (PD-1), the programmable cell death 1 (PD-L1) receptor ligand, and the programmable cell death 2 (PD-L2) ligand [31].

PD-1 (CD279) were isolated from cells which received by hybridization of T-cells mice and line of progenitor hematopoietic cells of the mouse. PD-1 is encoded by the Pdcd1 gene, which is located on chromosome 2 (2q37). The receptor belongs to the family of Type I receptors of the immunoglobulin superfamily (CD28) and is a transmembrane protein comprising an extracellular N-terminal IgV-like domain, a transmembrane domain, and a cytoplasmic tail. The molecular weight of the protein is $55 \mathrm{kD}$ and consists of 288 amino acids. In the cytoplasmic part of PD-1, there are two amino acid sequences, named tyrosine-based inhibitory motif and tyrosine-based switching motif. The amino acid sequence of the C-terminal tyrosine (TEYATIVF) is associated with proteintyrosine phosphatase 1 (SHP-1) and protein-tyrosine phosphatase 2 (SHP-2). Tyrosine phosphorylation and dephosphorylation of proteins are involved in key regulatory events of many signaling pathways leading to proliferation, differentiation, and death of cells [32, 33, 34, 35].

PD-1 is expressed on activated immune system cells such as CD4+ T-cells, CD8+ T- cells, B-cells, T-killers, monocytes, dendritic cells, and macrophages. Moreover, expression of PD-1 selectively increases in T-cells with long-acting antigens and is a marker of depleted $\mathrm{T}$ cells and cells with impaired effector function [36, 37].

Regulation of PD-1 expression is carried out in several ways [38]. Enhancement of expression of PD-1 on CD8+ T-cells and PD-L1 on cancer cells is associated with soluble factors, such as IL-6 and IL-10 [35,36]. In this case, the expression of PD-L1 on circulating monocytes positively correlates with the expression of PD-1 on any circulating CD4+ or CD8+ T-cells, indicating the possibility that the same factors increase the activity of both PD-1 and PD-L1. In addition, the authors found a significant increase in the expression of PD-1 on CD4+ and CD8+ Tcells in cancer tissues compared to cells from peripheral blood samples and normal gastric mucosa. These data suggest that the cancer cells themselves can influence the expression of PD-1 and PD-L1 in gastric cancer [38].

Another factor regulating the activity of expression of PD-1 may be the T-bet transcription factor.The role of T-bet transcription factor was revealedwhen studying the activation of antiviral CD8+ T-cells in chronic infections. The relationship between T-bet and PD-1 may depend on the state of the immune response against the viral infection. For example, the inverse correlation between PD-1 and T-bet during chronic infection was manifested only on the 15thday of the infection. In addition, adecrease in T-bet expression during an acute infection resulted in more pronounced expression of PD-1 on CD8+ T-cells. However, according to this author, the regulation of PD-1 by T-bet is not sufficient for the full expression of PD-1 during chronic infection. It is most likely that not only T-bet participates in the regulation of PD-1 but also other inhibitory receptors,persistent antigenic signals and other transcription factors engaged in the process [39].

The PD-1 gene contains two conserved CR-B and CR-C regions that affect the expression of the receptor and supersensitivity to DNase-I. CR-C contains the nuclear transcription factor of activated T-cells (NFAT), which plays an important role in the expression of Pdcd1. NFATc1 in CD4+ and CD8+ T-cells enhances the expression of PD-1 in the initial phases of antigen recognition by binding to CR-C and c-Fos to a site located in the CR-B site[31].

PD-L1 and PD-L2 are the two major ligands of PD-1. PD-L1 refers to the B7-CD28 immunoglobulin superfamily and is a transmembrane glycoprotein with a length of 290 amino acids [40]. The second PD-1 receptor ligand, the so-called B7-DC or PD-L2, is also a member of the B7 superfamily. Expression of mRNA encoding PDL1 was detected in all normal tissues of humans and mice. However, the expression and concentration of the PD-L1 protein itself on the cell surface were rare and were found only on the surface of macrophage-like cells of the liver, lungs, tonsils, and placenta tissue. The discrepancy between the expression of mRNA and the expression of cell protein indicates the important role of the posttranscriptional mechanism which controls PD-L1 expression. In general, expression of PD-L1 is regulated by both external and internal mechanisms [40].

The increase in synthesis of PD-L1 is largely due to external factors, such as anti-inflammatory cytokines. For example, interferon gamma can induce high levels of PD-L1 expression on the surface of normal epithelial, vascular endothelial and myeloid cells, as well as on the surface of T lymphocytes [41]. Induction of PD-L1 in numerous hematopoietic and non-homeopathic cells was reproducedin vitro conditions using several mediators associated with inflammatory processes. Gamma-interferon (IFN- $\gamma$ ) was the most effective mediator[42]. In addition,IFN- $\gamma$ can increase transcription of the receptor by binding to interferon regulatory factor 1, which is associated with two sites on the PD-L1 promoter to regulate expression of PD-L1 at the posttranscriptional level. [31]. Other inflammatory mediators are a vascular endothelial growth factor, colony stimulating factor of granulocytes and macrophages, that can influence the expression of PD-L1 [43]. 
Most cultured tumor cell lines derived from human or mice are expressingPD-L1on the surface of the cell. This receptor was detected by immunohistochemical analysis in many surgical and biopsy specimens of cancer. This fact implies that the microenvironment of cancer cells has the mechanism necessary to maintain PD-L1 expression. The minimal expression of PD-L1 in normal tissues and increased expression in tumor tissues is the unique feature of this protein. The selective expression of PD-L1 might be responsible for high antitumor efficacy and limited toxicity of blocking antibodies in clinical trials [44].

In addition,the PD-L1 protein was found in human oncogenic cells and 28 fibroblasts cell lines. The PDL1expression is due to T-cell IFN- $\gamma$ since it is the universal inducer of B7-H1. Interferon $\gamma$ is the main cytokine released by T-cells after recognition and activation of the antigen. The primary function of IFN- $\gamma$ is to enhance and maintain T-cell functions, such as increased regulation of histocompatibility molecules, enhancing the processing and presentation of antigen by target cells, or promoting $\mathrm{T}$-cell differentiation. Tumor cells use the natural physiological function of PD-L1, to protect themselves from an antitumor immune response, which is usually necessary for the protectionof tissue from increased immune activity in infection.It is believed that "adaptive resistance" plays an important role in the resistance of tumor cells to the T-cell attack in tumor microenvironment since this mechanism works specifically in the tumor region and even in the interaction of oncogenic cells and $\mathrm{T}$ lymphocytes [45].

\section{CONCLUSION}

Currently, the monoclonal antibodiesare widely used as antitumor target drugs in medicine. Immunological therapy of oncologic pathology is a set of the methods targeting enhancement and prolongation of immunological response,including the use of therapeutic vaccines, antibodies, cytokines, immune response inhibitors and adaptive administration of T-killers.Passive immunotherapy with the use of monoclonal antibodies directed against the target antigens of tumor cells is of interest for the therapy of oncological diseases. Monoclonal antibodies provide blockage of receptors associated with apoptosis of cells or inducing complement-dependent and antibody-dependent cellular cytotoxicity. The most traditional approach is the use of monoclonal antibodies against antigens specific only for tumor cells. In this regard, the main focus is the search for key antigens that will be neutralized by the $\mathrm{mAbs}$, leading to the complete elimination of the tumor without significant systemic side effects.

In present, monoclonal antibodies against the programmable cell death of PD-1 cells and its PD-L1 ligand have particular interest for targeted immunotherapy of cancer. The monoclonal antibodies Pembrolizumab and Nivolumab found the widestusage in the treatment of malignant melanoma. These drugs are laboratory-derived humanized antibodies against the PD1 receptor of cytotoxic lymphocytes. In addition to malignant melanoma, Pembrolizumab is effectively used against a number of oncological diseases, like lung cancer, colon cancer, ovarian cancer, etc. Under normal physiological conditions, the main function of the PD-1 receptor is to suppress the excessive activity of the immune system in peripheral tissues (autoimmune diseases) by apoptosis of T-cells. Tumor cells are capable of inhibiting anticancer immunity and they can evade cytotoxic T-cell attacks by negative regulation of PD-1 and PD-L1. The blockage of interaction between PD-1 and PD-L1 help to restore antitumor immunity and increase the secretion of cytokines. Some recent clinical trials of the of PD-1 and PD-L1 antibodies blockagealso demonstrated an effective response.

\section{Acknowledgments}

This work was funded by the project AP05130036 "Obtaining a strain of hybrid cells producing monoclonal antibodies against the programmed cell death receptor PD-1, a factor that reduces immunity against oncological diseases" within the budget program "Grant financing of scientific research" of the Ministry of Education and Science of Kazakhstan.

\section{REFERENCES}

1.Buzdin A.A., Zhavoronkov A.A., Borisov N.M. Personalizirovannyye podkhodisistemaprinyatiyaklinicheskogoresheniya $\mathrm{v}$ onkologiinaosnovaniianalizaaktivatsiisignal'nykhputey/The personalized approach and the system of clinical decision making in oncology based on the analysis of the activation of signaling pathways.Almanah.Innovacii $v$ onkologiil Almanac.Innovations in oncology, 2015, pp.1824.www.eafo.info | www.sk.ru.

2. Subburayalu J., Wilde B. "Programmed-cell-death"- Protein 1 (PD-1/CD279).Nephrologe, 2016, vol.11,pp. 70-72. doi: 10.1007/s11560-015-0037-y.

3. http://limbt.com/page/108/.

4. http://goods24.ru/onkologicheskie-zabolevaniya-v-kazaxstane/.

5.http://medinfo.kz/\#/dpsobl.

6.https://yvision.kz/post/361082. 
7.Riemer A.B., Hantusch B., Sponer B., Kraml G., Hafner C., Zielinski C.C., Scheiner O., Pehamberger H., Jensen-Jarolim E. High-molecular-weight melanoma-associated antigen mimotope immunizations induce antibodies recognizing melanoma cells.Cancer Immunology Immunotherapy, 2005, vol. 54,pp. 677-684. doi: 10.1007/s00262004-0632-7.

8.Hamanishi J., Mandai M., Matsumura N., Abiko K., Baba T., Konishi I. PD-1/PD-L1 blockade in cancer treatment: perspectives and issues.International Journal of Clinical Oncology,2016, vol. 21.pp. 462-473.doi: 10.1007/s10147-016-0959-z.

9. Wang J., Yuan R., Song W., Sun J., Liu D., Li Z. PD-1, PD-L1 (B7-H1) and Tumor-Site Immune Modulation Therapy: The Historical Perspective. Journal of Hematology \& Oncology,2017, vol. 10,pp. 34-42. doi: 10.1186/s 13045-017-0403-5.

10. Wang D., Guo L., Wu X. Checkpoint inhibitors in immunotherapy of ovarian cancer. Tumor Biology,2015, vol. 36,pp. 33-39. doi: 10.1007/s13277-014-2848-2.

11. Fujita K., Ikarashi H., Takakuwa K., et al. Prolonged disease-free period in patients with advanced epithelial ovarian cancer after adoptive transfer of tumor-infiltrating lymphocytes.Clinical Cancer Research,1995, vol. 1,pp. 501-507.PMID: 9816009.

12.Manjunath S.R., Ramanan G., Dedeepiya V.D., et al. Autologous immune enhancement therapy in recurrent ovarian cancer with metastases: A case report.Case Reports in Oncology,2012, vol. 5,pp. 114-118.doi: $10.1159 / 000337319$.

13. Spellman A., TangS.C. Immunotherapy for breast cancer: past, present, and future. Cancer Metastasis Review.,2016, vol. 35,pp. 525-546.doi: 10.1007/s10555-016-9654-9.

14.Mohit E., Hashemi A., Allahyari M. Breast cancer immunotherapy: monoclonal antibodies and peptidebased vaccines. Expert Review of Clinical Immunology, 2014, vol. 10,pp. 927-961. doi: 10.1586/1744666X.2014.916211.

15. Barrow C., Browning J., MacGregor D., et al. antigen expression in melanoma varies according to antigen and stage.Clinical Cancer Research, 2006, vol. 12,pp. 764-771.doi: 10.1158/1078-0432.CCR-05-1544.

16.Mittendorf E.A., Holmes J.P., Ponniah S., Peoples G.E. The E75 HER2/neu peptide vaccine.Cancer ImmunolImmunother,2008, vol. 57,pp. 1511-1521. doi: 10.1007/s00262-008-0540-3.

17. Peoples G.E., Holmes J.P., Hueman M.T., et al. Combined clinical trial results of a HER2/neu (E75) vaccine for the prevention of recurrence in high-risk breast cancer patients: U.S. Military Cancer Institute Clinical Trials Group Study I-01 and I-02. Clinical Cancer Research, 2008, vol. 14,pp. 797-803.doi: 10.1158/10780432.CCR-07-1448.

18.Niccolai E., Amedei A. Vaccine Immunotherapy Strategies in Colorectal Cancer Treatment.Single Cell Biology,2012, vol. 1,pp. 1-7. doi: 10.4172/2168-9431.1000102.

19. Menon A.G., Kuppen P.J., van der Burg S.H., et al. Safety of intravenous administration of a canarypox virus encoding the human wild-type p53 gene in colorectal cancer patients.Cancer Gene Therapy, 2003, vol. 10,pp. 509-517. doi: 10.1038/sj.cgt.7700600.

20. Kaufman H.L., Lenz H.J., Marshall J., Singh D., et al. Combination chemotherapy and ALVAC-CEA/B7.1 vaccine in patients with metastatic colorectal cancer.Clinical Cancer Research, 2008, vol. 14, pp.4843-4849. doi: 10.1158/1078-0432.CCR-08-0276.

21.Elkord E., Dangoor A., Burt D.J., Southgate T.D., et al. Immune evasion mechanisms in colorectal cancer liver metastasis patients vaccinated with TroVax (MVA-5T4). Cancer Immunology Immunotherapy, 2009, vol. 58,pp. 1657-1667. doi: 10.1007/s00262-009-0674-y.

22.Giantonio B.J., Catalano P.J., Meropol N.J., O’Dwyer P.J., Mitchell E.P., Alberts S.R. Bevacizumab in combination with oxaliplatin, fluorouracil, and leucovorin (FOLFOX4) for previously treated metastatic colorectal cancer: results from the Eastern Cooperative Oncology Group Study E3200.Journal of Clinical Oncology, 2007 , vol. 25, pp.1539-1544. doi: 10.1200/JCO.2006.09.6305.

23.Speetjens F.M., Kuppen P.J., Welters M.J., et al. Induction of p53-specific immunity by a p53 synthetic long peptide vaccine in patients treated for metastatic colorectal cancer.Clinical Cancer Research, 2009, vol. 15,pp. 1086-1095. doi: 10.1158/1078-0432.CCR-08-2227.

24.Dalerba P., Ricci A., Russo V., Rigatti D., et al. High homogeneity of MAGE, BAGE, GAGE, tyrosinase and Melan-A/MART-1 gene expression in clusters of multiple simultaneous metastases of human melanoma: implications for protocol design of therapeutic antigen-specific vaccination strategies.International Journal Cancer, 1998, vol. 17, no.77(2),pp. 200-204. PMID: 9650552.

25.Thara E., Barzi A. Immunotherapeutic Strategies for Colon Cancer: Monoclonal Antibody Therapy.Current Colorectal Cancer Reports,2015, vol. 11,pp. 84-91.doi: 10.1007/s11888-015-0260-y.

26. Mamalis A., Garcha M., Jagdeo J. Targeting the PD-1 pathway: a promising future for the treatment of melanoma.Archives of Dermatological Research,2014, vol. 306,pp. 511-519. doi: 10.1007/s00403-014-1457-7.

27. Shimizu T., Seto T., Hirai F., Takenoyama M., et al. Phase 1 study of pembrolizumab (MK-3475; anti-PD1 monoclonal antibody) in Japanese patients with advanced solid tumors.Investigational New Drugs.,2016, vol. 34,pp. 347-354.doi: 10.1007/s10637-016-0347-6.

28. Yamazaki N., Takenouchi T., Fujimoto M., et al. Phase 1b study of pembrolizumab (MK-3475; anti-PD-1 monoclonal antibody) in Japanese patients with advanced melanoma (KEYNOTE-041).Cancer Chemotherapy Pharmacology,2017, vol. 79,pp. 651-660. doi: 10.1007/s00280-016-3237-X. 
29. Francisco L.M., Sage P.T., Sharpe A.H. The PD-1 pathway in tolerance and autoimmunity.Immunology Review,2010, vol. 236,pp. 219-242. doi: 10.1111/j.1600-065X.2010.00923.x.

30. Barber D.L., Wherry E.J., Masopust D., Zhu B., et al. Restoring function in exhausted CD8 T cells during chronic viral infection.Nature, 2006, vol. 439,pp. 682-687.doi: 10.1038/nature04444.

31. Dong Y., Sun Q., Zhang X. PD-1 and its ligands are important immune checkpoints in cancer.Oncotarget,2017, vol. 8, no.2,pp. 2171-2186. doi: 10.18632/oncotarget.13895.

32. Ishida Y., Agata Y., Shibahara K., Honjo T. Induced expression of PD-1, a novel member of the immunoglobulin gene superfamily, upon programmed cell death.EMBO J.,1992, vol. 11,pp. 3887-3895.PMID: 1396582.

33. Shinohara T., Taniwaki M., Ishida Y., Kawaichi M., Honjo T. Structure and chromosomal localization of the human PD-1 gene (PDCD1).Genomics, 1994, vol. 23,pp. 704-706.doi: 10.1006/geno.1994.1562.

34. Starr R., Willson T.A., Viney E.M., et al.A family of cytokine-inducible inhibitors of signaling.Nature, 1997, vol. 387, pp. 917-921.doi: 10.1038/43206.

35. Lorenz U. SHP-1 and SHP-2 in T cells: two phosphatases functioning at many levels.ImmunologyReview.,2009, vol. 228, no.1, pp. 342-359. doi: 10.1111/j.1600-065X.2008.00760.x.

36.Agata Y., Kawasaki A., Nishimura H., Ishida Y., Tsubata T., Yagita H., Honjo T. Expression of the PD-1 antigen on the surface of stimulated mouse T and B lymphocytes.International Immunology, 1996, vol. 8,pp. 765772 .

37.Matsuzaki J., Gnjatic S., Mhawech-Fauceglia P., Beck A., et al. Tumor-infiltrating NY-ESO-1-specific CD8+ $\mathrm{T}$ cells are negatively regulated by LAG-3 and PD-1 in human ovarian cancer.Proceedings NationalAcademySciences USA,2010, vol. 107,pp. 7875-7880. doi: 10.1073/pnas.1003345107.

38. Saito H., Kuroda H., Matsunaga T., Osaki T., Ikeguchi M. Increased PD-1 expression on CD4+ and CD8+ $\mathrm{T}$ cells is involved in immune evasion in gastric cancer. Journal of Surgical Oncology,2013, vol. 107,pp. 517-522. doi: $10.1002 /$ jso. 23281 .

39. Kao C., Oestreich K.J., Paley M.A., Crawford A., et al. Transcription factor T-bet represses expression of the inhibitory receptor PD-1 and sustains virus-specific CD8+ T cell responses during chronic infection.Nature Immunology,2011, vol. 12,pp. 663-671. doi: 10.1038/ni.2046.

40.Sanmamed M.F., Chen L. Inducible expression of B7-H1 (PD-L1) and its selective role in tumor site immune modulation.Cancer Journal,2014, vol. 20,pp. 256-261. doi: 10.1097/PPO.0000000000000061.

41.Mazanet M.M.,Hughes C.C.W. B7-H1 is expressed by human endothelial cells and suppresses T cell cytokine synthesis.The Journal of Immunology,2002, vol. 169, pp.3581-3588.doi: 10.4049/jimmunol.169.7.3581.

42.Curiel T.J.,Wei S., Dong H., Alvarez X., Cheng P., et al. Blockade of B7-H1 improvesmyeloid dendritic cell-mediated antitumor immunity.NatureMedicine,2003, vol. 9,pp. 562-567.doi: 10.1038/nm863.

43.Sznol M., Chen L. Antagonist antibodies to PD-1 and B7-H1 (PD-L1) in the treatment of advanced human cancer-response.Clinical Cancer Research,2013, vol. 19,pp. 5542.doi: 10.1158/1078-0432.CCR-13-2234.

44.Hamid O., Robert C., Daud A., Hodi F.S., Hwu W-J., et al. Safety and tumor responses with lambrolizumab (anti-PD-1) inmelanoma.The New England Journal of Medicine,2013, vol. 369,pp. 134-144.doi: 10.1056/NEJMoa1305133.

45.Taube J.M., Anders R.A., Young G.D., Xu H., et al.Colocalization of inflammatory response with B7-h1 expression in human melanocytic lesions supports an adaptive resistance mechanism of immune escape.Science Translational Medicine,2012, vol. 4,pp. 127-137.doi: 10.1126/scitranslmed.3003689. 